\title{
62 Moderate endurance training and non-specific immunity
}

During roughly the first 20 minutes of moderate endurance training, there is an increase in natural killer cells (NK) as well as a minor rise in monocytes. The NK cells are not produced in higher numbers, rather larger amounts detach from the internal blood vessel walls. The acting stimulus here is the catecholamine effect which increases cardiac output. After the exertion is over, their number falls to below the original level and remains at a lower level for several hours. The NK cell count rises again at rest and remains constantly elevated in regular moderate-intensity endurance training.
The number of granulocytes, however, does not increase until after a good hour, whereas this increase does then remain constant for another 24 hours and the granulocytes' state of activity is elevated.

The benefits of non-specific immunity are particularly important in old age because they counteract the age-dependent fall in the specific-action T-lymphocytes.

Moderate endurance training is a good immunostimulant.

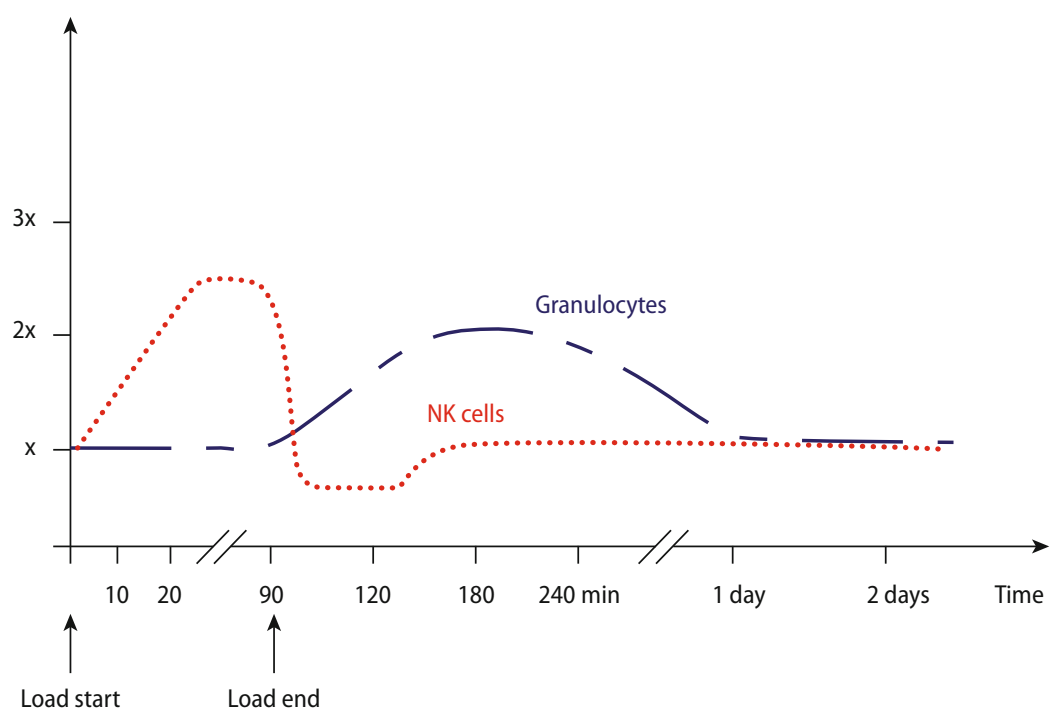

Fig. 62.1 Cell count changes in moderate endurance workout 